\title{
Cytokine Therapies in Neurological Disease
}

\author{
Shila Azodi ${ }^{1}$ (D) Steven Jacobson $^{1}$
}

Published online: 7 July 2016

(C) The American Society for Experimental NeuroTherapeutics, Inc. (outside the U.S.) 2016

\begin{abstract}
Cytokines are a heterogeneous group of glycoproteins that coordinate physiological functions. Cytokine deregulation is observed in many neurological diseases. This article reviews current research focused on human clinical trials of cytokine and anticytokine therapies in the treatment of several neurological disease including stroke, neuromuscular diseases, neuroinfectious diseases, demyelinating diseases, and neurobehavioral diseases. This research suggests that cytokine therapy applications may play an important role in offering new strategies for disease modulation and treatment. Further, this research provides insights into the causal link between cytokine deregulation and neurological diseases.
\end{abstract}

Key Words Cytokine · anticytokine $\cdot$ neurology $\cdot$ drug development.

\section{Introduction}

Cytokines are a heterogeneous group of glycoproteins that coordinate many physiological functions, including immune function, inflammation, hematopoiesis, homeostasis, and tissue repair. For example, cytokines regulate crosstalk between the innate and adaptive immune responses determining the magnitude of an immune response [1]. The deregulation of cytokines is observed in many neurological diseases, and, as such, their involvement in pharmacological therapies offers a promising avenue of research. However, there are complexities that make the

Steven Jacobson

jacobsons@ninds.nih.gov

1 Viral Immunology Section, National Institute of Neurological Disorders and Stroke, National Institutes of Health, Bethesda, MD 20892, USA prediction of pharmacological interventions difficult. First, it is still unclear whether cytokine deregulation is the cause or effect of these diseases. Second, depending on the context, cytokines can exhibit both inflammatory and anti-inflammatory effects. Third, these cytokines act through complex networks, and are involved in important homeostatic functions.

This review focuses on human clinical trials of cytokine and anticytokine therapies in a subset of neurological diseases. This research suggests that cytokine therapy applications may play an important role in offering new strategies for disease modulation and treatment. Further, this research provides insights into the causal link between cytokine regulation and neurological diseases.

Families of cytokines with similar structures and receptors allow overlapping and complementary action among cytokines. One method of cytokine classification is based on the similarities of their receptors with tumor necrosis factor (TNF), interleukin (IL)-1, and $\gamma \mathrm{C}$ superfamilies among the categories [2]. Other methods categorize cytokines by the primary cell of cytokine origin or functional role. In general, it is difficult to rely on a single classification system. The standard naming system for cytokines - in the order of their discovery-further reflects that individual cytokines can have multiple actions and receptors defying simple categorization.

Cytokines have been studied in neurological disease, contributing to both neuroprotective and neurodegenerative pathways. For example, granulocyte colony-stimulating factor (G-CSF) is a hematopoietic factor that has been found to act as a neural ligand to counteract programmed cell death. Supplementation of G-CSF has been used in stroke and amyotrophic lateral sclerosis (ALS) trials in an attempt to prevent neuronal loss. Alternatively, TNF family cytokines are proinflammatory with a myriad of actions as variable as hematopoietic cell proliferation and cell death. Anti-TNF therapies include monoclonal antibodies, as well as TNF receptor 
molecules that prevent TNF interaction with its receptors. Anti-TNF therapies have been used in inflammatory myopathies, Alzheimer's disease, and multiple sclerosis (MS). Unexpectedly, patients with MS had exacerbations of disease with anti-TNF therapy. The seemingly contradictory evidence from these trials has highlighted the importance of further studies into temporal patterns of cytokine expression in neurological disease and the nonlinear nature of cellular response to cytokines.

This article will review cytokine therapy trials in stroke, neuromuscular diseases, neuroinfectious diseases, demyelinating diseases, and neurobehavioral diseases. After a brief description of the neurological disease and current understanding of cytokine roles in the disease, we will summarize clinical trials to date targeting cytokines in these diseases.

\section{Cerebrovascular Disease}

\section{Stroke}

Stroke encompasses a broad category of disease defined as brain, spinal cord, or retinal cell death attributable to ischemia based on neuropathological, neuroimaging, and/or clinical evidence of permanent injury [3]. Acutely after stroke, levels of interferon (IFN)- $\gamma$ and TNF- $\alpha$ are decreased, and levels of IL1, IL-4, and IL-10 are increased [4, 5]. G-CSF is released by neurons in response to cerebral ischemia and can act to prevent neuron apoptosis and enhance vessel reformation in ischemic tissue [6].

Therapeutic trials have been designed to alter cytokine levels during acute stroke with the goal of reducing cerebral ischemia and improving clinical outcomes. IL-1 is a key mediator of inflammation and upregulates IL-6 in the cerebrospinal fluid (CSF) of stroke patients. IL-6 triggers inflammation by orchestrating leukocyte migration into brain parenchyma, elevation of body temperature, and in later stages tissue remodeling through astrogliosis and angiogenesis [7]. IL-1 receptor antagonist (anakinra) used in acute stroke was safe and well tolerated with trends in clinical improvement in patients with cortical infarcts. Secondary biological outcomes included reduced peripheral neutrophil count and lower IL-6 levels. In a placebo-controlled trial of patients with subarachnoid hemorrhage, anakinra infusion upon hospital admission was associated with lower IL-6 levels [5]. Small sample sizes and lack of trials with tissue plasminogen activator co-administration justify further exploration of this treatment option.

AXIS-2, a multicenter, randomized, placebo-controlled trial with 72-h infusion of G-CSF in patients with acute ischemic stroke did not find any benefit in clinical outcomes as measured by modified Rankin scale at 90 days. The treated patients not only had an expected rise in neutrophil count supporting therapeutic doses of G-CSF, but also had increased pulse and decrease in mean arterial pressure posited as possibly counteracting potentially beneficial effects that had been seen in preclinical models [8].

\section{Neuromuscular Disease}

\section{Autoimmune Inflammatory Myopathies}

The most common varieties of autoimmune inflammatory myopathies are dermatomyositis (DM), polymyositis (PM), and inclusion-body myositis (IBM). Pathologically, a humoral immune response against the vascular endothelium has been established in DM, while cytotoxic T-cell interactions with muscle have been shown in PM and IBM. While the antigens have not been identified and the role of associated antibodies is unclear, increased cytokine expression, including IL- $1 \alpha$, IL$1 \beta$, IFN- $\gamma$, and TNF- $\alpha$, are thought to be involved in the pathogenic process [9].

Therapies aimed at reducing the proinflammatory state in these myopathies have included several anticytokine drugs. Type 1 IFNs regulate cytotoxic T-cell activity and recruit Th1 cells to sites of inflammation. Sifalimumab, an anti-IFN- $\alpha$ monoclonal antibody, has been used in treatment of patients with DM and PM, resulting in reduction of multiple cytokines, including those that are inducible by type 1 IFNs, IL-2RA, monocyte chemoattractant protein-1, monocyte chemoattractant protein-2, and B-cell activating factor. Patients with at least $30 \%$ reduction in IL-2RA had more improvement in clinical outcomes of muscle motor testing [10].

TNF is a multifunctional proinflammatory cytokine with pleiotropic effects. In clinical trials of patients with DM, PM, and IBM in which the primary outcome measure was muscle strength, no benefit was seen with the TNF-blocking agents infliximab and etanercept. In another study, the use of infliximab in inflammatory myositis was associated with persistent cell infiltrate on muscle biopsy and significant increase in type 1 IFN activity [11]. How TNF blockade causes worsening inflammation in inflammatory conditions is poorly understood. Adding to the complexity of this story, 20 cases of DM or PM have been reported after induction of anti-TNF treatment for other autoimmune diseases [12].

Given the poor performance of TNF blockade in treatment of inflammatory myositis, alternative inflammatory pathways have been targeted for treatment. Despite cytotoxic $\mathrm{CD}^{+}$cell invasion and IL- $1 \alpha$ and IL-1 $\beta$ upregulation in IBM muscle, the disease is poorly responsive to steroids. IL-1 is produced by activated macrophages, directing differentiation and function of polarized innate and adaptive lymphoid cells. Anakinra use resulted in variable clinical benefit in muscle motor testing $[13,14]$. More specific targeting of IL-1 $\beta$ is underway with clinical trials using gevokizumab, an anti IL-1 $\beta$. 
Given the deregulation of cytokines in inflammatory myopathies, investigators have measured the effect of immunosuppressive treatments used in these diseases on cytokine levels. At diagnosis, IL-15 and its receptors were detected in mononuclear cells infiltrating muscle tissue of patients with DM and PM. The IL-15 level decreased with high-dose steroids followed by azathioprine, cyclophosphamide, or methotrexate, especially in patients with muscle motor testing improvements [15]. In a trial of rituximab in myositis, patients were stratified based on levels of type 1 IFN-regulated chemokine and cytokine scores. Patients with elevated scores responded to rituximab therapy and had a score reduction $[16,17]$.

\section{Amyotrophic Lateral Sclerosis (ALS)}

ALS is a neurodegenerative disease characterized by progressive loss of motor neurons leading to motor weakness and muscle atrophy. Several pathological abnormalities have been found in ALS, including genetic mutations, oxidative stress, mitochondrial dysfunction, and neuroinflammation [18]. ALS cytokine profiles have elevated IL-6, GM-CSF, IL-10, IL-2, and IL-15 levels compared with other neurological diseases [19]. As the etiology of disease is yet unknown, neuroprotective strategies have been implemented to alter disease progression.

As described previously, G-CSF can prevent neuronal apoptosis. Several small studies [20, 21] have demonstrated feasibility of G-CSF administration without significant change in clinical outcome measured by the ALS functional rating scale. Preliminary diffusion tensor imaging results suggested white matter tract preservation with treatment [21]. Another neuroprotective agent used in ALS has been epoetin alfa (EPO), regularly used in stimulation of red blood cell production. EPO receptors are found on neurons and astrocytes, and preclinical studies suggested EPO-mediated neuroprotection [22]. A large randomized trial of patients with ALS, involving 12 months of EPO treatment, resulted in no change in ALS functional rating scale score.

Another strategy in ALS clinical trials has been IL-1 blockade, which was shown in preclinical studies to slow the progression of neurodegeneration. In a recent 24-week trial of anakinra in patients with ALS, no change in clinical outcome was detected despite reduced levels of IL-6 and TNF [23].

\section{Neuroinfectious Disease}

\section{Human T-Lymphotropic Virus 1-Associated Myelopathy/Tropical Spastic Paraparesis (HAM /TSP)}

HAM/TSP is characterized by progressive lower extremity weakness, spasticity, and bladder/bowel sphincter dysfunction. The neuroinflammatory process linked to myelopathy is thought to begin with IFN- $\gamma$ secretion by infected CD ${ }^{+} \mathrm{T}$ cells in the CSF, stimulating $\mathrm{C}-\mathrm{X}-\mathrm{C}$ motif chemokine (CXCL)10 production by astrocytes. CXCL10, a chemokine promoting T-cell adhesion to endothelial cells and chemoattraction of inflammatory cells, increases mononuclear infiltration into the CSF creating a positive feedback loop for chronic inflammation in HAM/TSP [24]. In patients with HAM/TSP, levels of IFN- $\gamma$-producing T cells with loss of immune suppressive function have been shown to correlate with clinical severity [25]. IL-2 and IL-15 are deregulated in HAM/TSP and trigger spontaneous lymphoproliferation, increased signal transducer and activator of transcription 5 phosphorylation in lymphocytes, and increased frequency of virusspecific cytotoxic $\mathrm{CD}^{+}$T cells [26].

The virus-host interaction causing T-cell activation and maintenance of virus-specific T-cell stimulation is the target of IFN- $\alpha$ and IFN- $\beta$ 1a therapy in HAM/TSP. IFNs have cytostatic and antiviral properties that in short clinical trials were associated with clinical stability, reduction in virusspecific $\mathrm{CD}^{+} \mathrm{T}$ cells, and reduced spontaneous proliferation $[27,28]$. Targeted therapies in clinical trials next included antiTac, a monoclonal antibody that blocks the IL-2 receptor. Anti-Tac treatment was associated with decreased spontaneous lymphoproliferation and reduced proviral load [29]. Currently in trial in patients with HAM/TSP is Humik $\beta 1$, a humanized monoclonal antibody blocking IL-15 receptor $\beta$.

A novel approach to anticytokine therapy has been developed to target multiple cytokines. BNZ132-1-40 is a pegylated peptide that was designed to block the interface between the $\gamma \mathrm{C}$ receptor subunit of the IL-2 and IL-15 receptor and the respective cytokines [30]. In preclinical studies, it was shown the BNZ132-1-40 blocks both IL-2- and IL-15-induced signal transducer and activator of transcription 5 phosphorylation without effects on other $\gamma \mathrm{C}$ family cytokine function. Ex vivo HAM/TSP peripheral blood mononuclear cells showed decreased spontaneous proliferation when incubated with BNZ132-1-40 [26]. This novel approach to anticytokine therapy may be a promising therapeutic application in neurological and non-neurological disease in which multiple cytokines are pathogenic.

\section{Demeylinating Disease}

\section{Multiple Sclerosis (MS)}

MS is a neuroinflammatory disorder characterized clinically by transient neurological deficits corresponding to demyelinating lesions in the central nervous system (CNS), seen as contrast-enhancing lesions on magnetic resonance imaging. Histopathological findings of these lesions include deposition of inflammatory cytokines such as IL-12, IL-17, IL-23, and IFN- $\gamma$, in addition to T cells, B cells, antibodies, complement, and macrophages. Serum levels of IFN- $\gamma$ and TNF were 
elevated in patients with acute relapses, while IL-4, IL-10, and IL-15 expression was associated with resolution of inflammation [31]. This pattern is associated with a Th1 immune response, regularly seen in proinflammatory states of autoimmune disease and intracellular pathogens. Specifically, IL-12 and IL-23 are produced by inflammatory myeloid cells and play a role in the development of IL-17 producing [T helper (Th)17] cell responses.

Early disease-modifying therapies in MS aimed to shift the balance from a proinflammatory state to an anti-inflammatory and regulatory state with IFN- $\beta$. Patients treated with IFN- $\beta$ have decreased IL-17, IL-23 [32] IL-12, and IFN- $\gamma$ [33] levels, and increased IL-10 levels. Glatiramer acetate, a peptide-mimicking myelin basic protein, with similar efficacy to IFN- $\beta$, alters the cytokine profile in a similar manner [31, 34], especially in responders.

More targeted approaches in MS have included anti-IL-12 and anti-IL-23 blockade through a monoclonal antibody blocking their common subunit p40. IL-12 is associated with differentiation of naïve $\mathrm{CD} 4^{+} \mathrm{T}$ cells to $\mathrm{Th} 1$ cells with production of IFN- $\gamma$ and TNF- $\alpha$. IL-23 expands Th17 lymphocytes, promoting an inflammatory environment. Several clinical trials of the anti-IL12 and IL-23 have been well tolerated but not more efficacious than current therapies $[35,36]$.

Further anticytokine-specific interventions for MS have included the anti-IL-2 monoclonal antibody, daclizumab. The initial clinical trials with daclizumab were instrumental in revealing new mechanisms of IL-2 on the innate immune system, namely expansion of CD56 ${ }^{\text {bright }}$ natural killer cells [37]. In a 54-week trial of daclizumab in relapsing-remitting MS, $\mathrm{C}-\mathrm{C}$ chemokine receptor type 5 and chemokine (C-X-C motif) receptor 3, chemokines regulating leukocyte migration, expression was not altered, although levels of IL-12p40, a shared subunit for IL-12 and IL-23, was significantly decreased. Clinically, there was a reduction in contrast-enhancing lesions in these patients [38].

An example of an unanticipated physiologic effect of cytokine blockade occurred in the application of anti-TNF therapy in MS. TNF- $\alpha$ is a proinflammatory cytokine that has been shown to be elevated in the peripheral blood of patients with relapsingremitting MS [39], with elevated TNF- $\alpha$ mRNA expression in demyelinating plaques of patients with MS [40]. In mouse models, anti-TNF antibodies prevented the transfer of experimental autoimmune encephalomyelitis. The use of lenercept, a TNF-neutralizing molecule, in a clinical trial of patients with MS increased relapses compared with placebo. This led to investigation of dual nature of TNF that can be inflammatory or anti-inflammatory depending on patter of expression [41].

Another therapy that was first tested in and shown to ameliorate experimental autoimmune encephalomyelitis is transforming growth factor (TGF)- $\beta 2$. TGF- $\beta 2$ is a potent immunosuppressive cytokine reducing TNF expression and inhibiting cytotoxic T-lymphocyte generation. Eleven patients with chronic progressive MS had a reversible decline in glomerular filtration rate and anemia in a phase I trial of TGF- $\beta 2$. Although the trial was not designed to test clinical efficacy, several patients continued to have disease activity while on treatment [42].

In addition to targeting cytokines in MS, other diseasemodifying therapies alter cytokine profiles in patients with MS. Natalizumab, a monoclonal antibody against the integrin very late activation antigen, prevents activated T-cell entry into the CNS. In a trial of 31 patients with relapsing-remitting MS on natalizumab, comparison of pretreatment and posttreatment serum and CSF cytokine levels showed reduced CSF IL-1 $\beta$, IL-6, and IL-8, as well as chemokines CXCL9, CXCL10, and CXCL11. In particular, the peripheral levels of these proteins did not increase as one would expect from the known mechanism of natalizumab, suggesting an effect on peripheral T cells [43].

An important adjunct treatment in MS is vitamin D supplementation. As part of a studying evaluating the importance of the Th17 inflammatory response in MS, a placebo-controlled trial of high-dose vitamin D and stable IFN- $\beta$ treatment resulted in a statistically significant reduction in serum IL-17 levels [44]. Short- [45] and long-term [46] changes in cytokine profiles were seen in patients receiving glucocorticoid treatments for acute relapses.

\section{Neuromyelitis Optica (NMO)}

NMO is a neuroinflammatory disease characterized by demyelination in the optic nerves and spinal cord in the presence of aquaporin 4 antibodies. The underlying pathology is distinct from MS as the antibodies target astrocytes rather than oligodendrocytes. These antibodies have been shown to activate complement and lead to necrotic, destructive lesions. Initial therapeutic targets in NMO were against B cells given their role in antibody production. Recent efforts have helped to clarify the role of other immune cells in this process, including Th17 cells in producing these destructive lesions [47]. IL-6, which promotes antibody production in activated B cells as well as Th17 differentiation, was present in elevated levels in the serum and CSF has been shown to be elevated in patients with NMO, especially during relapse [48].

Patients with NMO who failed treatments, including azathioprine and rituximab, were enrolled in pilot study [49] and reviewed in a retrospective case study [50] of tocilizumab, an anti-IL-6 humanized monoclonal antibody. Tocilizumab treatment decreased relapse rates and was associated with better recovery from relapses. Scores for pain, which can be a challenging manifestation of NMO disease, were significantly lower on the tocilizumab. Tocilizumab is approved for use in rheumatoid arthritis and is a promising targeted therapy in NMO. 


\section{Neurobehavioral Disease}

\section{Alzheimer's Disease (AD)}

$\mathrm{AD}$ is a common neurodegenerative dementia characterized by memory impairment and neuropsychiatric complications. The etiology is as yet unknown, although hallmarks of the disease include pathological findings of intracellular neurofibrillary tangles and extracellular amyloid plaques. This chronic deposition of amyloid has been associated with microglial activation and proinflammatory cytokine production, including IL-1 $\beta$, TNF- $\alpha$, IL-6, and IL-18 [51]. Studies to date seek to clarify whether the inflammatory cytokines are a response to plaque formation, the initial insult produces plaque formation, or a combination of these events $[52,53]$.

A phase II randomized clinical trial of subcutaneous etanercept in $\mathrm{AD}$ established that this drug is well tolerated in a 24-week study, although there were no changes in cognitive, behavioral, or global function measured over this period [54]. Previously, an open-label pilot study enrolled 15 patients with $\mathrm{AD}$ in a 6-month open-label perispinal administration of etanercept [55]. Results included significant improvements in standardized cognitive testing. These findings are important given that etanercept does not penetrate the blood-brain barrier when administered systemically. A randomized trial of perispinal administration of etanercept is currently ongoing.

The effect of commonly used treatments in $\mathrm{AD}$ on cytokine profiles has also been investigated. Acetylcholinesterase inhibitors and omega-3 fatty acids have not shown significant effects on IL-6 or TNF- $\alpha$ levels in the blood $[56,57]$. Conflicting data presented in studies of cytokine levels in AD may reflect different stages of disease and possible importance of CSF cytokine studies.

\section{Conclusion}

To date, the approach to cytokine therapy in neurological disease has been to block cytokines found to be elevated in disease states and supplement cytokines that are found to have possible neuroprotective or anti-inflammatory effects. Trials with variable clinical benefit, such as anti-TNF in inflammatory myopathies, suggest we need more information on cytokine profiles for appropriate patient selection. Additionally, clinical outcome measures and timing of therapy in diseases with chronic progression requires attention. These clinical trial findings highlight the complex activity and expression of cytokines in neurological diseases. Longitudinal data on cytokine expression profiles will enhance our understanding of the role of cytokines, as well as help us optimize pharmacological intervention in these diseases.

Further research into the relationship between serum and CSF cytokine profiles will advance our therapeutic applications of systemic cytokine and anticytokine treatments in neurological disease. Although the application of etanercept in $\mathrm{AD}$ was only carried out in a small pilot trial, the difference in response to peripheral and perispinal application is important to investigate. The disconnect between CSF and peripheral cytokine expression changes in patients with MS on natalizumab contributes to questions about the role of the blood-brain barrier in peripheral cytokine actions on the CNS.

Cytokine therapy applications in neurological diseases will play an important role in offering new strategies for disease modulation and treatment. New developments on the horizon, such as BNZ132-1-40, provide promising alternatives to monoclonal antibodies in blocking cytokine subsets. As our understanding of neurological disease mechanisms improves, we will be able to offer novel directed therapies that repurpose currently available cytokine drugs and tailor combinations of these drugs. The field of cytokine therapy in neurological disease holds promise in offering patients new options and treatments.

\section{Compliance with Ethical Standards}

Required Author Forms Disclosure forms provided by the authors are available with the online version of this article.

\section{References}

1. Belardelli F, Ferrantini M. Cytokines as a link between innate and adaptive antitumor immunity. Trends Immunol 2002;23:201-208.

2. McInnes, Iain (2013). Cytokines in Firestein GS, et al (Ed.) Kelley's Textbook of Rheumatology. (367-377). Philadelphia, PA: Elsevier.

3. Sacco RL, Kasner SE, Broderick JP, et al. An updated definition of stroke for the 21st century: a statement for healthcare professionals from the American Heart Association/American Stroke Association. Stroke 2013;44:2064-2089.

4. Benarroch EE. Autonomic-mediated immunomodulation and potential clinical relevance. Neurology 2009;73:236-242.

5. Singh N, Hopkins SJ, Hulme S, et al. The effect of intravenous interleukin-1 receptor antagonist on inflammatory mediators in cerebrospinal fluid after subarachnoid haemorrhage: a phase II randomised controlled trial. J Neuroinflammation 2014 11:1.

6. Schabitz WR, Laage R, Vogt G, et al. AXIS: a trial of intravenous granulocyte colony-stimulating factor in acute ischemic stroke. Stroke 2010;41:2545-2551.

7. Erta M, Quintana A, Hidalgo J. Interleukin-6, a major cytokine in the central nervous system. Int J Biol Sci 2012;8:1254-1266.

8. Ringelstein EB, Thijs V, Norrving B, et al. AXIS 2 Investigators. Granulocyte colony-stimulating factor in patients with acute ischemic stroke: results of the AX200 for Ischemic Stroke trial. Stroke 2013;44:2681-2687.

9. Mastaglia FL, Garlepp MJ, Phillips BA, et al. Inflammatory myopathies: clinical, diagnostic and therapeutic aspects. Muscle Nerve 2003;27:407-425.

10. Guo X, Higgs BW, Rebelatto M, et al. Suppression of soluble T cell-associated proteins by an anti-interferon- $\alpha$ monoclonal antibody in adult patients with dermatomyositis or polymyositis. Rheumatology (Oxford) 2014;53:686-695. 
11. Dastmalchi M, Grundtman C, Alexanderson H, et al. A high incidence of disease flares in an open pilot study of infliximab in patients with refractory inflammatory myopathies. Ann Rheum Dis 2008;67:1670-1677.

12. Brunasso AM, Aberer W, Massone C. New onset of dermatomyositis/polymyositis during anti-TNF- $\alpha$ therapies: a systematic literature review. ScientificWorldJournal 2014;2014: 179180

13. Kosmidis ML, Alexopoulos H, Tzioufas AG, et al. The effect of anakinra, an IL1 receptor antagonist, in patients with sporadic inclusion body myositis (sIBM): a small pilot study. J Neurol Sci 2013;334:123-125.

14. Zong M, Dorph C, Dastmalchi M, et al. Anakinra treatment in patients with refractory inflammatory myopathies and possible predictive response biomarkers: a mechanistic study with 12 months follow-up. Ann Rheum Dis 2014;73:913-920.

15. Zong M, Loell I, Lindroos E, et al. Effects of immunosuppressive treatment on interleukin-15 and interleukin-15 receptor $\alpha$ expression in muscle tissue of patients with polymyositis or dermatomyositis. Ann Rheum Dis 2012;71:1055-1063.

16. Reed AM, Crowson CS, Hein M, et al. Biologic predictors of clinical improvement in rituximab-treated refractory myositis. BMC Musculskelet Disord 2015;16:257.

17. López De Padilla CM, Crowson CS, Hein MS, et al. Interferonregulated chemokine score associated with improvement in disease activity in refractory myositis patients treated with rituximab. Clin Exp Rheumatol 2015;33:655-663.

18. Moujalled D, White AR. Advances in the development of diseasemodifying treatments for amyotrophic lateral sclerosis. CNS Drugs 2016;30:227-243.

19. Mitchell RM, Freeman WM, Randazzo WT, et al. A CSF biomarker panel for identification of patients with amyotrophic lateral sclerosis. Neurology 2009;72:14-19.

20. Chiò A, Mora G, La Bella V, et al. Repeated courses of granulocyte colony-stimulating factor in amyotrophic lateral sclerosis: clinical and biological results from a prospective multicenter study. Muscle Nerve 2011;43:189-195.

21. Duning T, Schiffbauer H, Warnecke T, et al. G-CSF prevents the progression of structural disintegration of white matter tracts in amyotrophic lateral sclerosis: a pilot trial. PLOS ONE 2011;6: e17770.

22. Lauria G, Dalla Bella E, Antonini G et al. Erythropoietin in amyotrophic lateral sclerosis: a multicentre, randomised, double blind, placebo controlled, phase III study. J Neurol Neurosurg Psychiatry 2015;86:879-886.

23. Maier A, Deigendesch N, Müller K et al. Interleukin-1 antagonist anakinra in amyotrophic lateral sclerosis - a pilot study. PLOS ONE 2015;10:e0139684.

24. Ando H, Sato T, Tomaru U, et al. Positive feedback loop via astrocytes causes chronic inflammation in virus-associated myelopathy. Brain 2013;136:2876-2887.

25. Yamano Y, Araya N, Sato T, Utsunomiya A, et al. Abnormally high levels of virus-infected IFN-gamma + CCR4+ CD4+ CD25+ T cells in a retrovirus-associated neuroinflammatory disorder. PLOS ONE 2009;4:e6517.

26. Massoud R, Enose-Akahata Y, Tagaya Y, et al. Common $\gamma$-chain blocking peptide reduces in vitro immune activation markers in HTLV-1-associated myelopathy/tropical spastic paraparesis. Proc Natl Acad Sci U S A 2015;112:11030-11035.

27. Izumo S, Goto I, Itoyama Y, et al. Interferon-alpha is effective in HTLV-I-associated myelopathy: a multicenter, randomized, doubleblind, controlled trial. Neurology 1996;46:1016-1021.

28. Oh U, Yamano Y, Mora CA, et al. Interferon-betala therapy in human T-lymphotropic virus type I-associated neurologic disease. Ann Neurol 2005;57:526-534.
29. Lehky TJ, Levin MC, Kubota R, et al. Reduction in HTLV-I proviral load and spontaneous lymphoproliferation in HTLV-I-associated myelopathy/tropical spastic paraparesis patients treated with humanized anti-Tac. Ann Neurol 1998;44:942-947.

30. Nata T, Basheer A, Cocchi F, et al. Targeting the binding interface on a shared receptor subunit of a cytokine family enables the inhibition of multiple member cytokines with selectable target spectrum. J Biol Chem 2015;290:22338-51.

31. Oreja-Guevara C, Ramos-Cejudo J, Aroeira LS, et al. Th1/Th2 cytokine profile in relapsing-remitting multiple sclerosis patients treated with glatiramer acetate or natalizumab. BMC Neurol 2012;12:95.

32. Kürtüncü M, Tüzün E, Türkoğlu R, et al. Effect of short-term interferon- $\beta$ treatment on cytokines in multiple sclerosis: significant modulation of IL-17 and IL-23. Cytokine 2012;59:400-402.

33. Graber JJ, Ford D, Zhan M, et al. Cytokine changes during interferon-beta therapy in multiple sclerosis: correlations with interferon dose and MRI response. J Neuroimmunol 2007;185:168174.

34. Tumani H, Kassubek J, Hijazi M, et al. Patterns of TH1/TH2 cytokines predict clinical response in multiple sclerosis patients treated with glatiramer acetate. Eur Neurol 2011;65:164-169.

35. Vollmer TL, Wynn DR, Alam MS, et al. A phase 2, 24-week, randomized, placebo-controlled, double-blind study examining the efficacy and safety of an anti-interleukin-12 and -23 monoclonal antibody in patients with relapsing-remitting or secondary progressive multiple sclerosis. Mult Scler 2011;17:181-191.

36. Segal BM, Constantinescu CS, Raychaudhuri A, et al. Repeated subcutaneous injections of IL12/23 p40 neutralising antibody, ustekinumab, in patients with relapsing-remitting multiple sclerosis: a phase II, double-blind, placebo-controlled, randomised, doseranging study. Lancet Neurol 2008;7:796-804.

37. Bielekova B. Daclizumab therapy for multiple sclerosis. Neurotherapeutics 2013;10:55-67.

38. Bielekova B, Howard T, Packer AN, et al. Effect of anti-CD25 antibody daclizumab in the inhibition of inflammation and stabilization of disease progression in multiple sclerosis. Arch Neurol 2009;66:483-489.

39. Larochelle C, Alvarez JI, Prat A. How do immune cells overcome the blood- brain barrier in multiple sclerosis? FEBS Lett 2011;585: $3770-3780$.

40. Bitsch A, Kuhlmann T, Da Costa C, et al. Tumour necrosis factor alpha mRNA expression in early multiple sclerosis lesions: correlation with demyelinating activity and oligodendrocyte pathology. Glia 2000;29:366-375.

41. Luchtman, DW, Ellwardt E, Larochelle C, et al. IL-17 and related cytokines involved in the pathology and immunotherapy of multiple sclerosis: current and future developments. Cytokine Growth Factor Rev 2014;25:403-413.

42. Calabresi PA, Fields NS, Maloni HW, et al. Phase 1 trial of transforming growth factor beta 2 in chronic progressive MS. Neurology 1998;51:289-292.

43. Mellergård $\mathrm{J}$, Edström $\mathrm{M}$, Vrethem $\mathrm{M}$, et al. Natalizumab treatment in multiple sclerosis: marked decline of chemokines and cytokines in cerebrospinal fluid. Mult Scler 2010;16:208-217.

44. Toghianifar N, Ashtari F, Zarkesh-Esfahani SH, et al. Effect of high dose vitamin D intake on interleukin-17 levels in multiple sclerosis: a randomized, double-blind, placebo-controlled clinical trial. J Neuroimmunol 2015;285:125-128.

45. Mirowska-Guzel DM, Kurowska K, Skierski J, et al. High dose of intravenously given glucocorticosteroids decrease IL-8 production by monocytes in multiple sclerosis patients treated during relapse. $\mathrm{J}$ Neuroimmunol 2006;176:134-140.

46. Mirowska-Guzel D, Gromadzka G, Kurowska K, et al. Long-term effect of high doses glucocorticosteroids on mRNA expression for 
IL-6 and IL-8 in relapsed multiple sclerosis patients. Immunopharmacol Immunotoxicol 2010;32:416-421.

47. Varrin-Doyer M, Spencer CM, Schulze-Topphoff U, et al. Aquaporin 4-specific $\mathrm{T}$ cells in neuromyelitis optica exhibit a Th17 bias and recognize clostridium ABC transporter. Ann Neurol 2012;72:53-64.

48. Uzawa A, Mori M, Arai K, et al. Cytokine and chemokine profiles in neuromyelitis optica: significance of interleukin-6. Mult Scler 2010;16:1443-1452

49. Araki M,Matsuoka T,Miyamoto K, et al. Efficacy of the anti-IL-6 receptor antibody tocilizumab in neuromyelitis optica: a pilot study. Neurology 2014;82:1302-1306.

50. Ringelstein M, Ayzenberg I, Harmel J, et al. Long-term therapy with interleukin 6 receptor blockade in highly active neuromyelitis optica spectrum disorder. JAMA Neurol 2015;72:756763.

51. Wen-Ying Wang, Meng-Shan Tan, Jin-Tai Yu, et al. Role of proinflammatory cytokines released from microglia in Alzheimer's disease. Ann Transl Med 2015;3:136.
52. Morimoto K, Horio J, Satoh H, et al. Expression profiles of cytokines in the brains of Alzheimer's disease (AD) patients compared to the brains of non-demented patients with and without increasing AD pathology. J Alzheimers Dis 2011;25:59-76.

53. Griffin WS. Neuroinflammatory cytokine signaling and alzheimer's disease. N Engl J Med 2013;368:770-771.

54. Butchart J, Brook L, Hopkins V, et al. Etanercept in Alzheimer disease: a randomized, placebo-controlled, double-blind, phase 2 trial. Neurology 2015;84:2161-2168

55. Tobinick E, Gross H, Weinberger A, et al. TNF-alpha modulation for treatment of Alzheimer's disease: a 6-month pilot study. MedGenMed 2006;8:25.

56. Richardson C, Gard PR, Klugman A, et al. Blood pro-inflammatory cytokines in Alzheimer's disease in relation to the use of acetylcholinesterase inhibitors. Int J Geriatr Psychiatry 2013;28:1312-317.

57. Freund-Levi Y, Hjorth E, Lindberg C, et al. Effects of omega-3 fatty acids on inflammatory markers in cerebrospinal fluid and plasma in Alzheimer's disease: the OmegAD study. Dement Geriatr Cogn Disord 2009;27:481-490. 